\section{Meiotic chromosome pairing and recombination take refuge in the telomeres}

\section{Rayasandram Muniyappa Ranganath}

In their recent Review article, Barzel and Kupiec $^{1}$ analyse some important issues concerning how homologous DNA sequences pair to facilitate homologous recombination (HR). However, the models discussed for meiosis are only relevant to organisms that form bivalents (yeasts, flies, worms, plants and vertebrates). Enlarging the focus to include organisms with novel meiotic formats would help to build a holistic view of not only pairing and HR events but also how these processes relate to chromosome segregation, genetics, sex and evolution.

Plants with permanent translocation heterozygosity (listed in REF. 2) are ideal organisms for such studies. In Oenothera lamarckiana (evening primrose), which is discussed here as a typical representative of this group, all chromosomes are connected end to end in a ring during meiosis I (MI). Despite the lack of homology between parental chromosomes owing to translocations, MI is successfully executed by segregating alternate chromosomes in the ring (parental sets) to opposite poles; the two parental sets, known as Renner complexes, are inherited as a single linkage unit that is unaltered through generations ${ }^{3}$. Because pairing is restricted to chromosome ends, telomeres emerge as the exclusive sites (hot spots) of both pairing and recombination. It would be immensely rewarding to examine the nature of meiotic chromosome pairing in the ring in the light of the models described by Barzel and Kupiec ${ }^{1}$.
In eukaryotes, $\mathrm{HR}$ is credited with two important functions during meiosis: it promotes genetic diversity through allele shuffling and also ensures proper chromosome segregation by forming chiasmata ${ }^{4}$. At least one chiasma per bivalent is necessary to allow segregation of homologues to opposite poles during $\mathrm{MI}^{5,6}$. In evening primrose $(2 \mathrm{n}=14)$, a minimum of 14 chiasmata is necessary to form an MI ring ${ }^{2,3}$ (as opposed to 1 for each of the 7 possible bivalents). Regular formation of a chromosome ring implies that telomeric regions in evening primrose are competent to sustain robust pairing and HR events, leading to proper MI chromosome segregation. Studying these events would shed light on how end-to-end pairing is regulated to ensure that parental chromosomes are positioned alternately in the ring and also segregated to opposite poles.

From a genetics perspective, sex is synonymous with meiotic $\mathrm{HR}^{7}$. Despite their sequence diversity ${ }^{8}$, plant telomeres are not known to possess genes. The crossovers that occur in telomeric regions of evening primrose are therefore neutral, in that they do not generate genetic diversity. Lack of genetic recombination might lead to the accumulation of deleterious mutations over generations (Muller's ratchet; see REF. 7); by the same token, however, this feature also protects the Renner complexes from breaking apart. How the ring formers deal with these unique situations would be an interesting focus for evolutionary genetics.
The novel format in which crossover takes place in evening primrose eliminates the genetic and evolutionary consequences of allele shuffling, but retains its mechanistic functions for chromosome segregation through chiasma. Meiosis as a process may have survived, but without generating genetic diversity. Consequent production of 'clones through meiosis' effectively converts sexual reproduction into an asexual one, lending support to the 'twofold cost of sex' hypothesis (see REF. 9) in a unique way. The same situation exists in Rhoeo spathacea (boat lily), another well-known ring former with $2 \mathrm{n}=12$ (see REF. 10).

Molecular dissection of the telomeres of evening primrose and other ring formers, using an 'omics' approach, should enrich our understanding of telomeres and also illuminate the poorly defined but fascinating complexities of meiotic pairing and HR.

Cytogenetics and Developmental Biology Laboratory, Department of Botany, Bangalore University, Jnanabharathi campus, Bangalore 560056, India.

e-mail: rayasran@rediffmail.com

1. Barzel, A. \& Kupiec, M. Finding a match: how do homologous sequences get together for recombination? Nature Rev. Genet. 9, 27-37 (2008).

2. Levy, M. \& Levin, D. A. Genic heterozygosity and variation in permanent translocation heterozygotes of the Oenothera biennis complex. Genetics 79 493-512 (1975).

3. Cleland, R. E. Oenothera: Cytogenetics and Evolution (Academic, London, 1972).

4. Cromie, G. A. \& Smith, G. R. Branching out: meiotic recombination and its regulation. Trends Cell Biol. 17 448-455 (2007)

5. Mezard, C., Vignard, J., Drouaud, J, \& Mercier, R. The road to crossovers: plants have their say. Trends Genet. 23, 91-99 (2007).

6. Nicklas, R. B. Chromosome segregation mechanisms. Genetics 78, 205-213 (1974).

Hillis, D. M. Asexual evolution: can species exist without sex? Curr. Biol. 17, R543-R544 (2007)

8. Lamb, J. C., Yu, W. Han, F. \& Birchler, J. A. Plant chromosomes from end to end: telomeres, heterochromatin and centromeres. Curr. Opin. Plant Biol. 10, 116-122 (2007).

9. Agrawal, A. F. Evolution of sex: why do organisms shuffle their genotypes? Curr. Biol. 16, R696-R704 (2006).

10. Ranganath, R. M. \& Umesh, T. G. Meiosis-I in Rhoeo spathacea - a contemporary view. Beitr. Biol. Pflanzen 70, 21-40 (1997). 